\title{
ELECTRONIC LEVEL STRUCTURE OF GROUP III HEAVY ELEMENT CLUSTERS STUDIED BY PHOTOELECTRON SPECTROSCOPY
}

\author{
M. GAUSA, G. GANTEFÖR, H.O. LUTZ and K.H. MEIWES-BROER \\ Fakultät für Physik, Universität Bielefeld, 4800 Bielefeld 1, (F.R.G.)
}

\begin{abstract}
UV-photoelectrons of mass-defined indium and thallium cluster anions have been analyzed at a detachment energy of $3.68 \mathrm{eV}$ in the size range $n=2-20$. The photoelectron threshold energies are in rough agreement with the values predicted by the metal droplet model. A number of low-lying excited states of the neutral-clusters are detected. The high atomic $s$-orbital binding energies appear to induce alkali-like character of $\mathrm{In}_{n}^{-}$and $\mathrm{Tl}_{n}^{-}$.
\end{abstract}

\section{INTRODUCTION}

The electronic level structure of clusters is directly accessible by techniques which combine photoelectron spectroscopy with intense cluster sources. Due to the mostly sloppy structure of clusters it is highly desirable to at least fix their mass; hence, charged species are generally employed. When starting with negatively charged ground state clusters, photodetachment probes transitions into ground and excited neutral states in the geometry of the anion. This method has only recently been applied to gas-phase elemental clusters [1-7] and provided electron affinities and energetic positions of low-lying excited states. Although theory is still lagging behind experiment, a number of physical properties can already be extracted from such photoelectron (PE) spectra. Several theoretical approaches, such as the model of an electron gas confined to the volume of the cluster (jellium model [8-10]), or quantum chemical calculations [e.g. 11-13] give at least a rough reproduction of the photodetachment thresholds.

In this contribution we concentrate on clusters of heavy elements of the main Group III. With increasing atom mass the atomic $s / p$ separation increases. Consequently, the low-energy cluster levels of $\mathrm{In}_{n}$ and $\mathrm{Tl}_{n}$ should arise from the atomic $p$-orbital, in contrast to those of, for example, aluminium clusters. 


\section{EXPERIMENTAL}

Experimental details have been discussed previously $[5,6]$. In short, metal cluster anions are produced in a laser vaporization source without additional ionization. After cooling in a (pulsed) supersonic jet the anions are massseparated in a time-of-flight (TOF) mass spectrometer collinearly to the source direction. Under ultrahigh vacuum conditions, fixed-frequency light of an ultraviolet laser $\left(\mathrm{N}_{2}\right.$-laser, $\left.h v=3.68 \mathrm{eV}\right)$ detaches electrons from massidentified anion bunches. The electron energies are analyzed by recording their flight times in a magnetic-bottle TOF electron spectrometer [14] perpendicular to the cluster beam direction. The electron energy resolution, $\Delta E / E$, depends on a variety of experimental parameters, in particular the magnetic field configuration, the photoionization volume and the primary cluster anion's velocity. In the spectra presented here $\Delta E / E \gtrsim 0.05$ was achieved. Monomer anion photoelectron spectra serve to calibrate the electron spectrometer.

\section{RESUITS AND DISCUSSION}

\section{Mass spectra}

Before photodetachment, the ion beam composition is studied by time-offlight analysis. Figure 1 depicts mass spectra of $\mathrm{In}_{n}^{-}$and $\mathrm{Tl}_{n}^{-}$. These spectra show similar intensity distributions to those of a variety of other metal cluster anions and ions produced in this source: Basically, broad distributions without significant outstanding lines appear which reflect the minor role of individual binding energies during cluster growth. The $\mathrm{In}_{n}^{-}$clusters are partly contaminated; this indicates the necessity of good mass separation. We note a slightly enhanced intensity for $n=13$; recall that also for $\mathrm{Al}_{n}^{-}$an intensity step can be produced at $n=13$ by detuning the source from "uniform spectrum" to "structured spectrum" conditions (see Fig. 2 as an example). The spectrum in Fig. 2 has been taken at higher resolution (using a time-offlight reflectron); steps and/or strong lines appear at $n=7,13,23,28,42,47$, 50,55 , etc. So far it is not clear whether the jellium model is applicable for trivalent clusters [15] or if the bonding retains substantial covalent character as has been calculated [11]. Nevertheless, for $\mathrm{Al}_{n}$ there are some experimental findings beyond simple mass spectroscopy (unimolecular decay [16], PE spectroscopy [5,17], reactive etching [18], or photoionization [19]) which can

Fig. 1. Time-of-flight (TOF) mass spectra of (a) indium and (b) thallium cluster anions produced directly (i.e. without additional ionization) in a laser vaporization source. The ion bunches have an energy of $300 \mathrm{cV}$ and are formed by grid switching. 


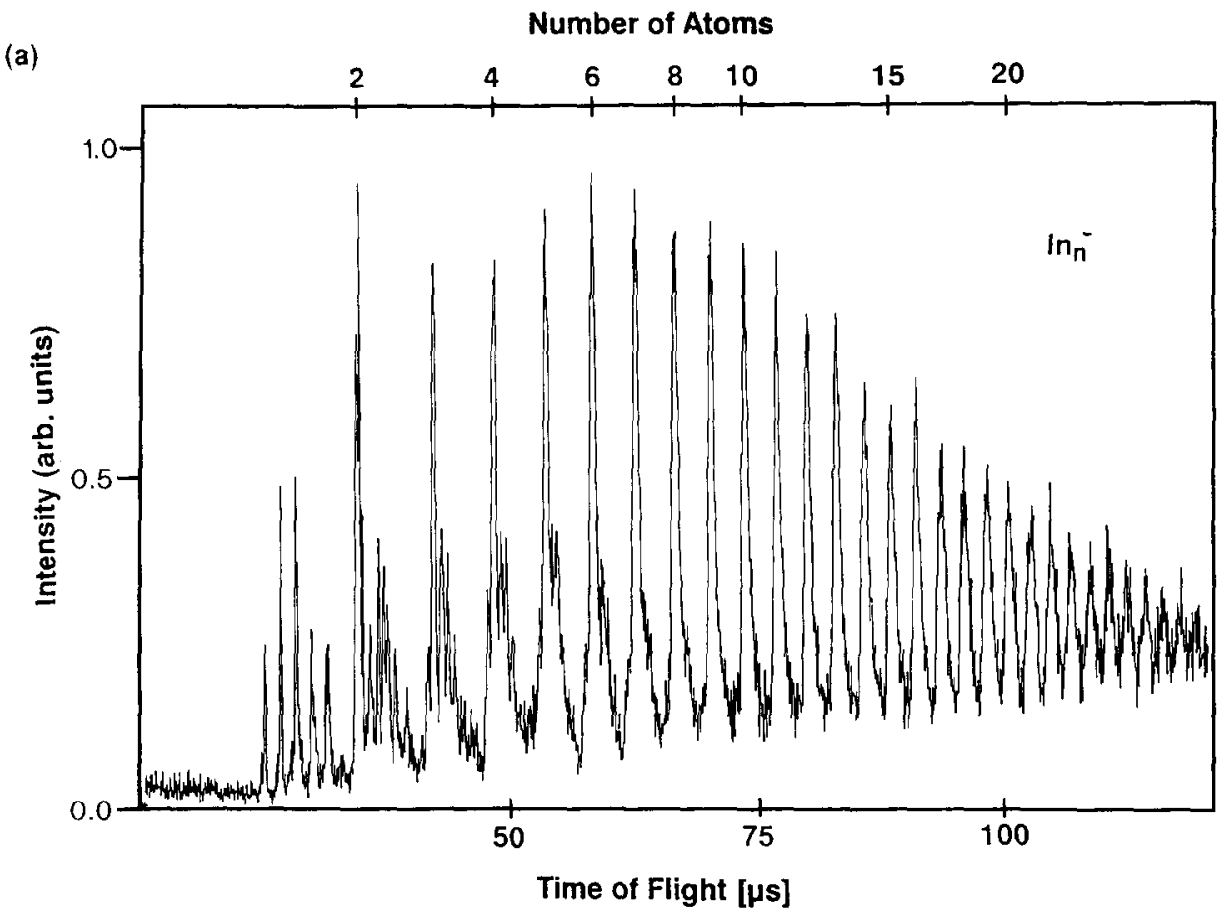

(b)

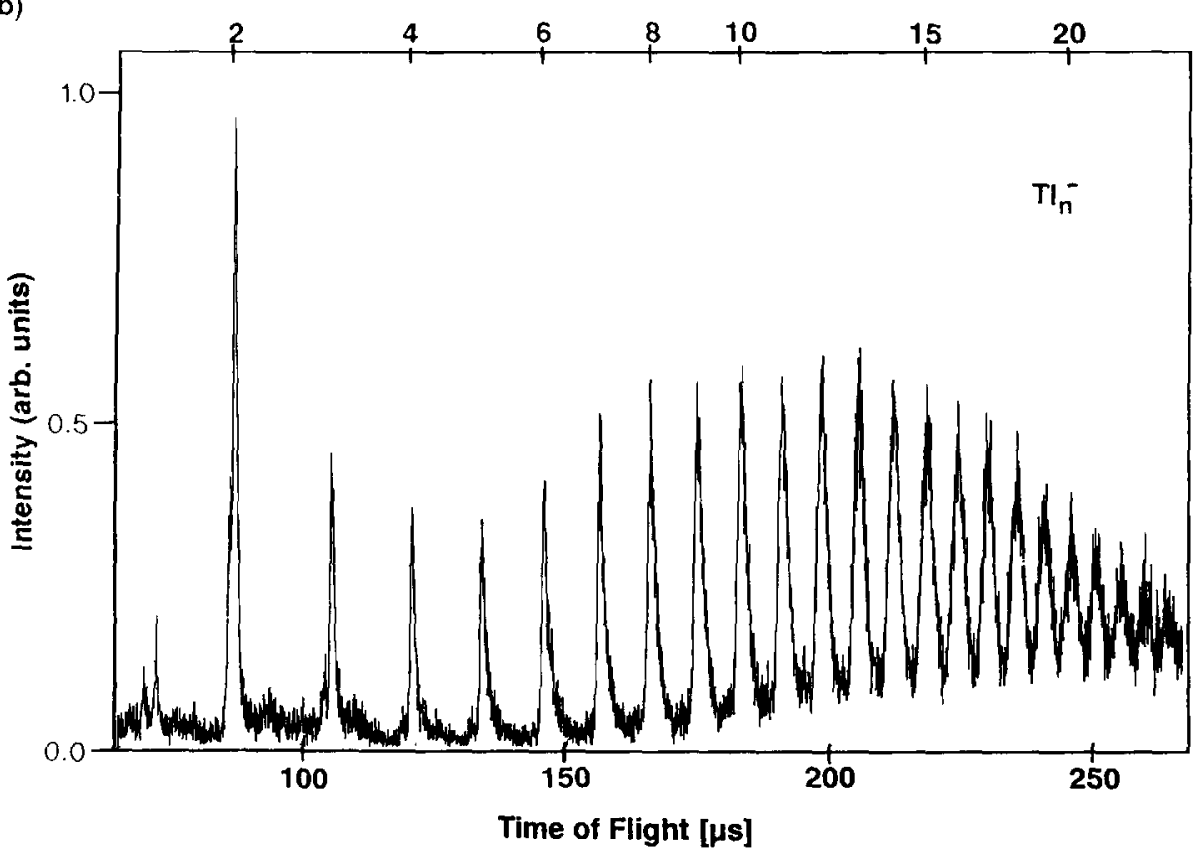


Number of Atoms

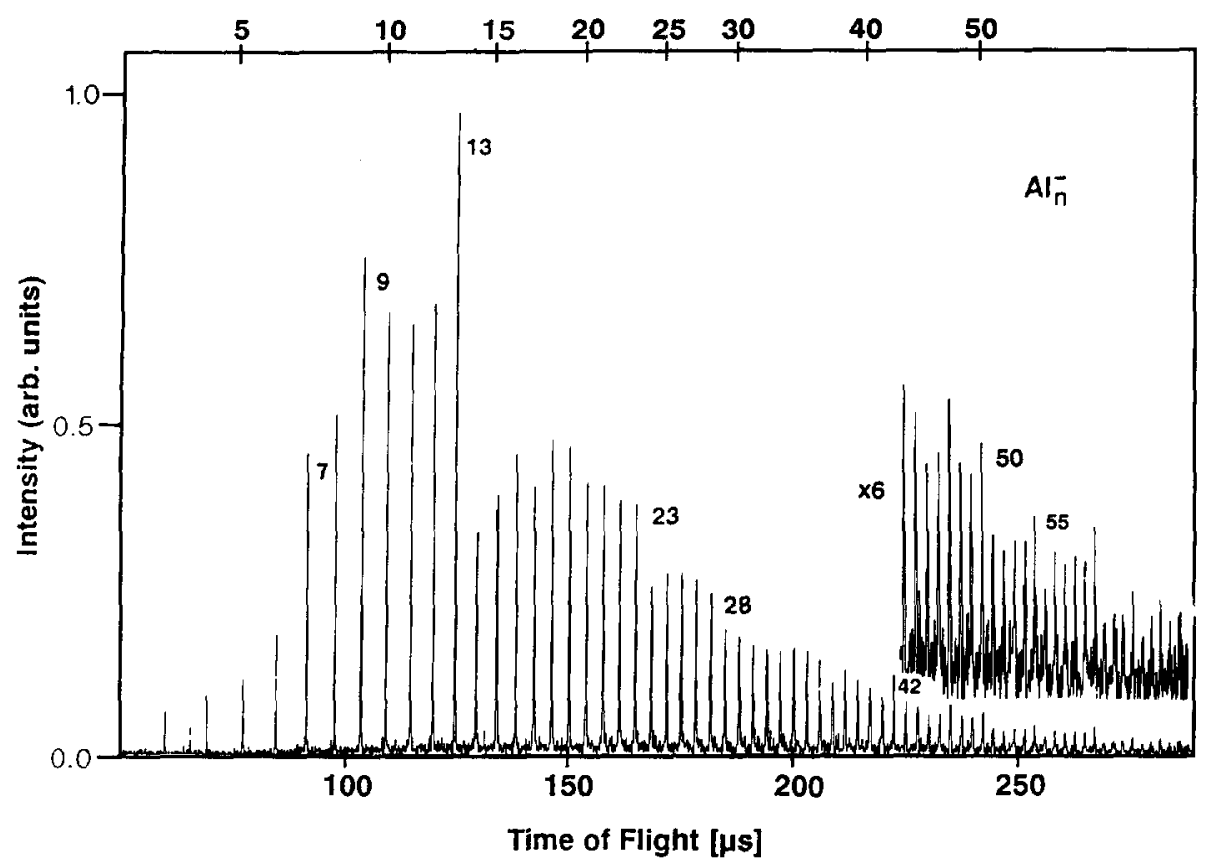

Fig. 2. TOF mass spectrum of aluminium cluster anions, now taken in a TOF reflectron. The source parameters are tuned to "structured spectrum" conditions in order to demonstrate the shell behaviour of $\mathrm{Al}_{n}^{-}$.

partly be explained by the shell model. When going to $\mathrm{In}_{n}$ or $\mathrm{Tl}_{n}$ the atomic valency should decrease as the atomic $s / p$ separation increases. Consequently, intensity structures in mass spectra are expected to be different, possibly weaker, if compared with the $\mathrm{Al}_{n}$ case. Indeed, the $\mathrm{In}_{n}^{-}$and $\mathrm{Tl}_{n}^{-}$mass spectra cannot be significantly influenced by tuning the source conditions to "structured spectrum" conditions. The slightly enhanced (reproducible) intensities of $\mathrm{In}_{13}^{-}$and $\mathrm{Tl}_{13}^{-}$coincide with the shell closing for 40 electrons ( $2 p$-level). Mass spectra of positively charged $\operatorname{In}_{n}[20]$ tend to exhibit pronounced intensities at $n=7,14$, and 23 as known from, for example, sputtered $\mathrm{Al}_{n}^{+}$[21]; again, for $\mathrm{In}_{n}^{+}$these structures are not as distinct as for $\mathrm{Al}_{n}^{+}$and are subject to changes in ionization conditions.

\section{Photoelectron spectra}

Pulsed UV laser light ( $h v=3.68 \mathrm{eV}, \approx 0.5 \mathrm{~mJ}$ per pulse) is utilized to detach electrons from mass-identified anion bunches. In Fig. 3 we present the PE spectra of $\mathrm{In}_{n}^{-}$cluster anions in the size range of 2-15 atoms, and in Fig. 


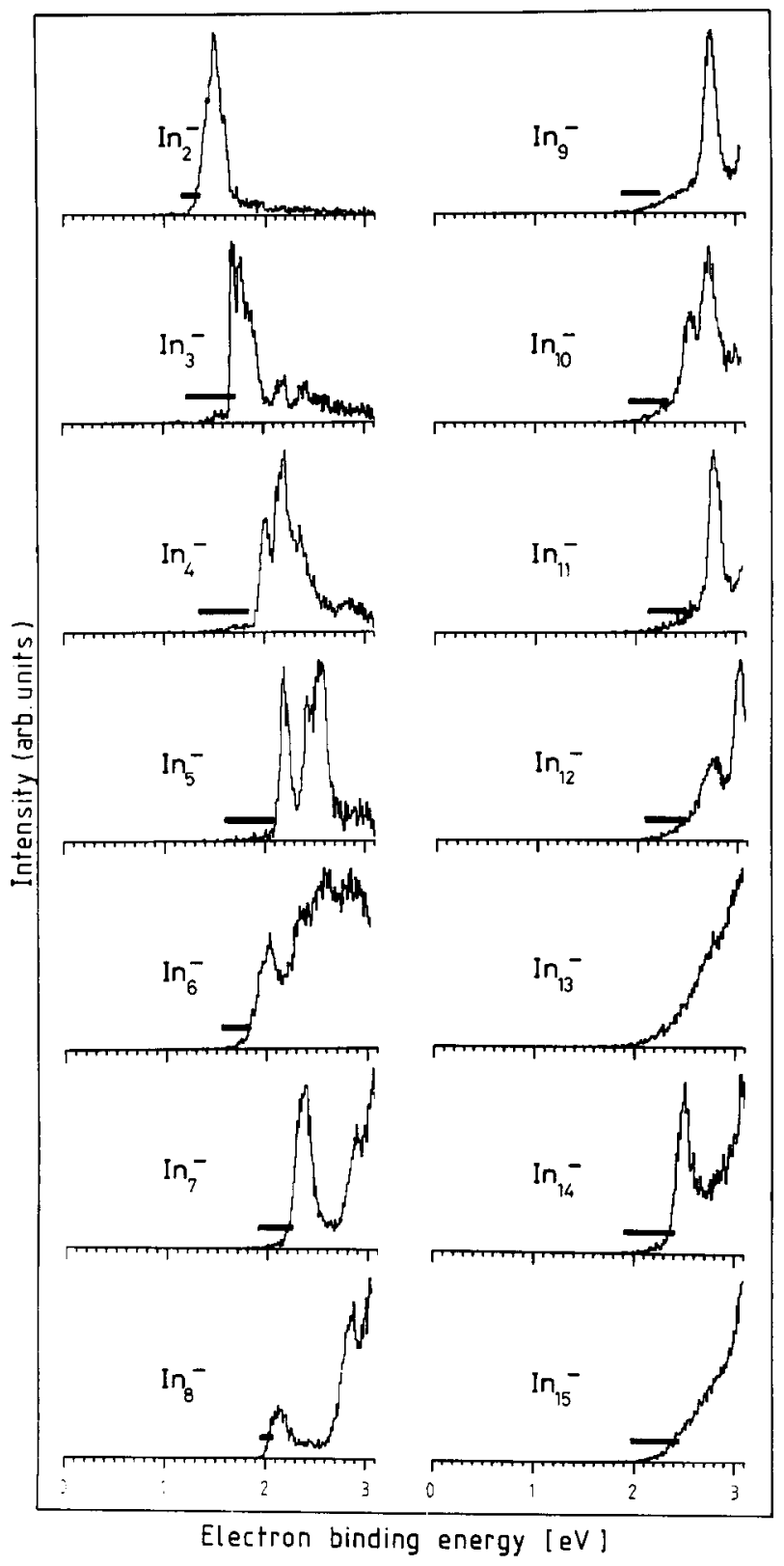

Fig. 3. Photoelectron spectra of $\mathrm{In}_{n}^{-}$clusters $(n=2-15)$ at a detachment energy of $3.68 \mathrm{eV}$ $(337 \mathrm{~nm})$. Each spectrum is an average over $10^{4}$ shots. No background signal correction has been performed. Photodetachment thresholds $\left(E_{\mathrm{TH}}\right)$ as estimates of the upper limits of adiabatic electron affinities are marked by horizontal solid bars. 


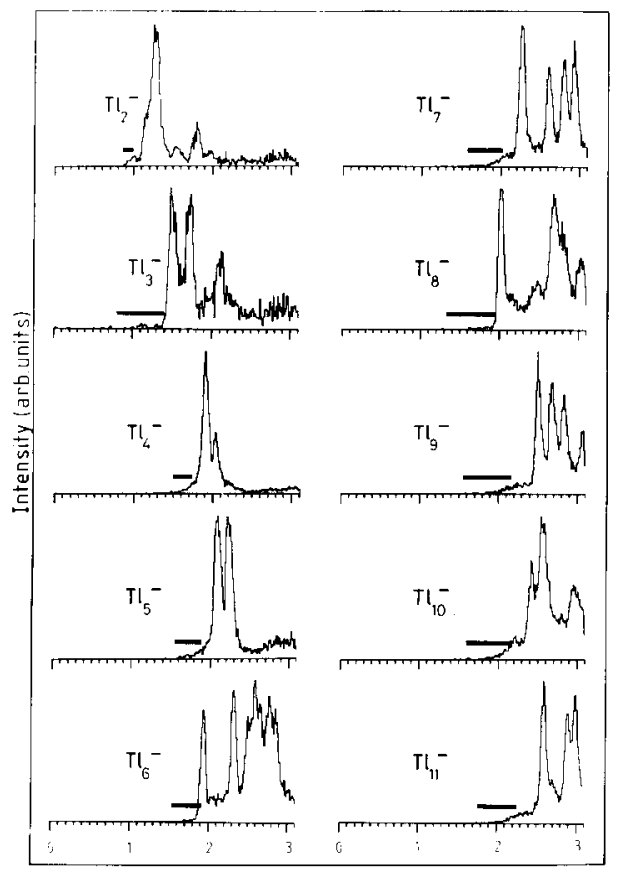

Electron binding energy [eV]

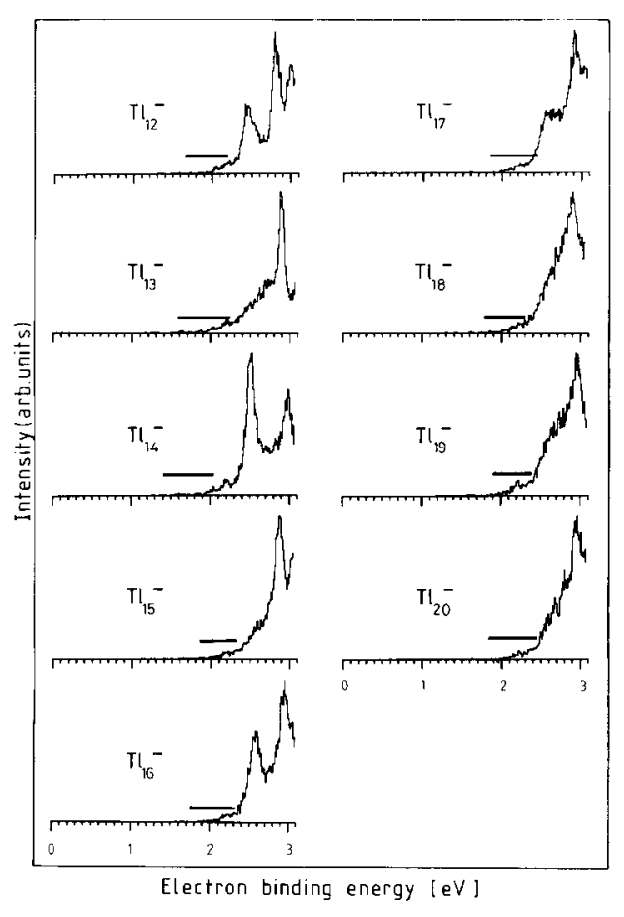

Fig. 4. Photoelectron spectra of $\mathrm{Tl}_{n}^{-}$clusters $(n=2-20)$.

4 those of $\mathrm{Tl}_{n}^{-}, n=2-20$. The data displayed are a result of a numerical conversion of experimental electron TOF spectra. The vertical axis represents full scale photoelectron count (uncorrected for spectrometer efficiency, etc.) so that the intensities do not directly represent the detachment cross-sections. As usual for beam experiments, the energy scale is normalized to the vacuum level; in this case the data directly give the electron binding energy $E_{\mathrm{B}}$, i.e. the photon energy less the kinetic energy of the detached electrons. Photodetachment induces a vertical transition from the initial anionic (ground) state into the neutral ground state or into excited neutral states. In the frozen orbital picture, the measured PE spectra image the orbital energies. This approximation (Koopman's theorem) is of course not sufficient to describe the situation, especially in the case of metals. Here, correlation and relativistic effects have to be considered as well as relaxation processes after detachment. Electron intensity at lowest $E_{\mathrm{B}}$ corresponds to a transition into the lowest neutral state with an appropriate spin multiplicity and geometry; this gives the photodetachment threshold energy $E_{\mathrm{TH}}$ (marked by bars). We may regard $E_{\mathrm{TH}}$ as an estimate of the upper bound of the adiabatic electron affinity.

We define the onset of the electron signal above background (fixed at $1.5 \%$ of maximum intensity) corrected by the instrumental broadening to be the 
PE-threshold $E_{\mathrm{TH}}$. Several electron spectra show an "unsharp" threshold behaviour, as will be discussed below; in some cases the threshold energy determination depends more on intensity considerations than on the instrumental resolution. The onset uncertainties (see bar width and Table 1) vary with the cluster size and are derived from the slopes of the electron spectra near $E_{\mathrm{TH}}$. At present, more elaborate methods of the threshold deter-

\section{TABLE 1}

Photoelectron threshold energies $E_{\mathrm{TH}}$ (as estimates of upper bonds of adiabatic electron affinities) of indium and thallium cluster anions (in $\mathrm{eV}$ )

\begin{tabular}{|c|c|c|}
\hline$n$ & $\mathrm{In}_{n}^{-}$ & $\mathrm{Tl}_{n}^{-}$ \\
\hline 2 & $1.27 \pm 0.1$ & $0.95_{-0.10}^{+0.05}$ \\
\hline 3 & $1.5 \pm 0.25$ & $1.1 \pm 0.3$ \\
\hline 4 & $1.7_{-0.35}^{+0.15}$ & $1.65_{-0.15}^{+0.1}$ \\
\hline 5 & $1.9_{-0.3}^{+0.2}$ & $1.75_{-0.2}^{+0.15}$ \\
\hline 6 & $1.75_{-0.2}^{+0.1}$ & $1.73 \pm 0.2$ \\
\hline 7 & $2.1+0.15$ & $1.87_{-0.3}^{+0.15}$ \\
\hline 8 & $2.04_{-0.1}^{+0.05}$ & $1.66_{-0.35}^{+0.3}$ \\
\hline 9 & $2.05 \pm 0.2$ & $1.87 \pm 0.32$ \\
\hline 10 & $2.13 \pm 0.2$ & $1.88_{-0.28}^{+0.25}$ \\
\hline 11 & $2.34_{-0.25}^{+0.15}$ & $2.01 \pm 0.25$ \\
\hline 12 & $2.28_{-0.2}^{+0.2}$ & $2.05_{-0.4}^{+0.15}$ \\
\hline 13 & & $1.94 \pm 0.3$ \\
\hline 14 & $2.2_{-0.3}^{+0.2}$ & $2.00_{-0.4}^{+0.05}$ \\
\hline 15 & $2.21 \pm 0.25$ & $2.17_{-0.30}^{+0.15}$ \\
\hline 16 & & $2.17_{-0.4}^{+0.15}$ \\
\hline 17 & & $2.2_{-0.35}^{+0.25}$ \\
\hline 18 & & $2.1_{-0.3}^{+0.2}$ \\
\hline 19 & & $2.15_{-0.25}^{+0.25}$ \\
\hline 20 & & $2.13 \pm 0.3$ \\
\hline
\end{tabular}


mination are not useful as the physical origin of the near-threshold spectral shapes is unclear. In particular, if there is no distinct maximum in the spectrum, any threshold determination will to a large extent depend on the accumulated number of counts and will, therefore, be fairly arbitrary (e.g. $\left.\mathrm{In}_{13}\right)$.

Spectral features beyond $E_{\mathrm{TH}}$ belong to transitions into excited neutral cluster states in the geometry of the anion. Obviously, many clusters exhibit several low-lying excited states, see, for example, $\mathrm{Tl}_{7}^{-}$or $\mathrm{Tl}_{9}^{-}$. All structures remain unchanged when the laser light flux is changed by a factor of ten. Multiphoton effects or photoneutralization of photofragments can thus largely be excluded.

\section{Threshold behaviour}

Like PE spectra of all other metal clusters, the data on $\mathrm{In}_{n}^{-}$and $\mathrm{Tl}_{n}^{-}$show a gradual shift of $E_{\mathrm{TH}}$ with increasing number of atoms $n$. In both systems, $E_{\mathrm{TH}}$ at the largest $n$ is still about $2 \mathrm{eV}$ below the corresponding polycrystalline bulk work function. This is in agreement with the metal sphere model [22] according to which the difference between EA and the bulk work function depends linearly on the inverse sphere radius. A comparison of various cluster PE data to this model has been published recently $\left(\mathrm{Cu}_{n}^{-}\right.$[1], $\mathrm{Al}_{n}^{-}$[5], $\mathrm{Ni}_{n}^{-}, \mathrm{Ag}_{n}, \mathrm{Sn}_{n}^{-}$ [6], and $\mathrm{Pd}_{n}^{-}$[23]). For $\mathrm{In}_{n}^{-}$and $\mathrm{Tl}_{n}^{-}$, we find a rough agreement of $E_{\mathrm{TH}}$ with the values predicted by the classical picture (see Fig. 5); the data of $\mathrm{Al}_{n}^{-}$are also included. The metal sphere model, however, is not seriously expected to work in the particle size range investigated here. Recent variation density calculations within a spherical jellium reproduce the measured threshold trends, including the systematic deviation from the $1 / R$ dependence [24].

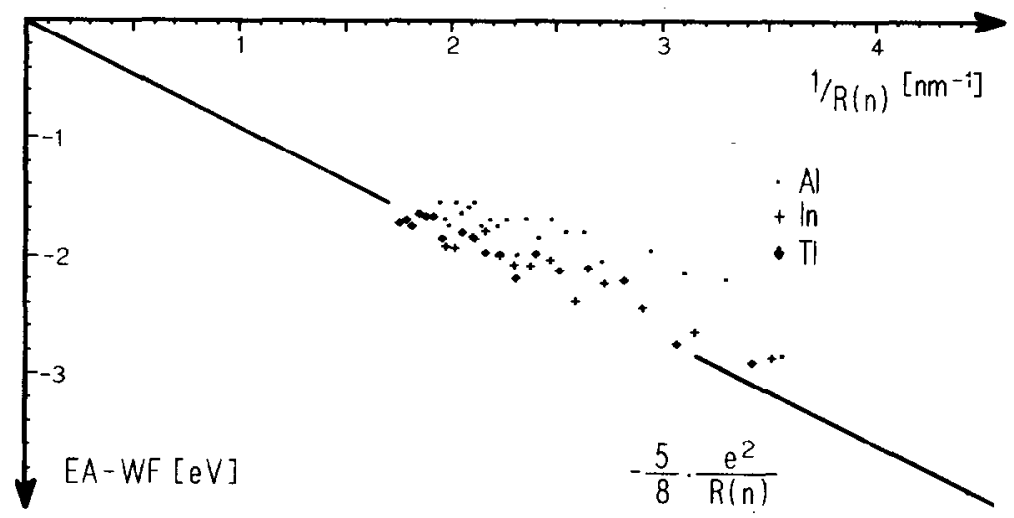

Fig. 5. Difference between experimental photodetachment threshold energies $E_{\mathrm{TH}}$ (as estimates of upper bonds of electron affinities) and bulk work function (WF) versus the reciprocal cluster radius, $R$, according to the metal sphere model [6,22]. 
This result should not be taken as a proof of good electrical conductivity of the cluster. Indeed, the electron confinement to volumes less than $10 \AA$ in diameter introduces discrete energy states, with separations of the order of $1 \mathrm{eV}$. These states are also reflected in the peak structure of the PE spectra. Instead, the agreement might be an indication of the degree of electron delocalization; note in particular that $\mathrm{Ni}_{n}^{-}$and $\mathrm{Pd}_{n}^{-}$(their bulk densities of states near the Fermi level are determined by localized $d$-electrons) strongly disagree with the classical model $[6,23]$.

An unsolved problem of $\mathrm{In}_{n}^{-}$and $\mathrm{Tl}_{n}^{-}$is the origin of low-energy tails in the PE-spectra. Especially for $\mathrm{Tl}_{n}^{-}$they may extend as far down as about $1 \mathrm{eV}$. Their intensity is extremely low and is fairly independent of the source conditions. The exception is $\mathrm{Tl}_{3}^{-}$. Upon source detuning, the tail grows into a pronounced peak which might originate from an excited anion state. Note that the spectra result from mass-identified clusters; when tuning the firing time of the detachment laser between two mass lines, the electron signals, including the flat tails, vanish. Therefore the tails open a wide field of speculation; one explanation might be the coexistence of jellium-like (spherical or spheroidal) clusters and covalently bound ones in which the charge is strongly localized (e.g. on a corner site); for such "isomers" with localized charge, the electron affinities should show only a weak $n$-dependence. For further discussion, we neglect these low-intensity tails.

\section{Electronic level structure}

The electron spectra of $\mathrm{In}_{n}^{-}$and $\mathrm{Tl}_{n}^{-}$show a number of similar features. For example, the line groups gradually shift to higher energies for $n=2-5$, and at $n=6$ a low-energy peak appears; another low-energy peak is observed at $n-8$, followed by a significant gap, etc. There are also differences between the spectra of the different systems. For example, the single line of $\mathrm{In}_{9}^{-}$is replaced by three narrow lines in the case of $\mathrm{Tl}_{9}^{-}$; the line widths of $\mathrm{Tl}_{n}^{-}$are throughout narrower than those of $\mathrm{In}_{n}^{-}$.

The strong similarity sheds some light on the origin of the observed features. Group III elements are characterized by $s^{2} p^{1}$ configuration yielding ${ }^{2} P$ atomic ground states. The $s$-orbital binding energies increase with increasing nuclear charge, resulting in higher $s \rightarrow p$ excitation energies for the heavy elements (In: $4.3 \mathrm{eV}$; T1: $5.6 \mathrm{eV}$ [25]) compared with aluminium (Al: $3.6 \mathrm{eV}$ ). In spite of the different $s$-orbital binding energies, the line positions for $\operatorname{In}_{n}^{-}$and $\mathrm{Tl}_{n}^{-}$at the same $n$ are very similar. We may therefore conclude that mainly the atomic $p$-orbitals contribute to the spectra of Figs. 3 and 4. This finding is in agreement with expectation. For small $\mathrm{Al}_{n}$, ab initio calculations demonstrate substantial covalent character [11]. Bulk Al is well described by the nearly-free 
electron model. The atomic levels hybridize into the $s p$ bands of the solid, resulting in a broad bulk PE spectrum. Therefore, we expect $s-p$ merging for $\mathrm{Al}_{n}$ in a certain $n$-range. Indeed, recent $\mathrm{PE}$ work on $\mathrm{Al}_{n}$ gives the first (although weak) hints to a band gap closing somewhere between $n=10$ and 30 [17].

Another criterion for a gap closing might be the agreement with the jellium model, each atom donating three electrons to the electron gas. In this picture, the $\mathrm{Al}_{n} s-p$ gap should already be closed at $n=7$ (for positively charged clusters) as the unimolecular decay of sputtered $\mathrm{Al}_{n}^{+}[16,21]$ shows a clear shell effect already at $n=7$.

Following the same line of reasoning for $\mathrm{In}_{n}^{-}$and $\mathrm{Tl}_{n}^{-}$, we only find a very weak ion intensity enhancement at $n=13$ (see above) which could also arise from a geometric shell closing. The PE spectra show no clear evidence of shell effects under the assumption of atomic trivalency.

A promotion of the atomic $p$-electrons into a "conduction band" and a strong $s$-electron localization should lead to an alkali-like situation. Disregarding flat $\mathrm{PE}$ thresholds and concentrating on the first strong maxima in Figs. 3 and 4 , we find a pronounced cven-odd oscillation for $n \gtrsim 4$. In addition, a significant drop in the first line binding energy occurs at $n=8$, which is accompanied by a large low-intensity area (gap). The same situation is found in copper $[1,2]$ and silver [6,23] PE spectra where it can be explained by the distorted jellium model $[9,26]$. Therefore, our measurements may provide evidence for an (to a certain extent) alkali-like character of $\operatorname{In}_{n}^{-}$and $\mathrm{Tl}_{n}^{-}$in the investigated size range.

\section{CONCLUSION}

The strong similarity of $\mathrm{In}_{n}^{-}$and $\mathrm{Tl}_{n}^{-} \mathrm{PE}$ spectra manifests their similar electronic character, which is different from that of $\mathrm{Al}_{n}^{-}$. The high $s-p$ separation of the heavy Group III metal atoms prevents an $s / p$ band closing in the observed size range. The high $s$-orbital binding energy appears to induce alkali-like character of $\mathrm{In}_{n}^{-}$and $\mathrm{Tl}_{n}^{-}$, as the first strong lines in the PE spectra exhibit a pronounced even-odd behaviour as well as a clear "shell effect" at $n=8$. In addition, the PE threshold energies nicely follow the metal droplet model; this is another hint of strong electron delocalisation in $\mathrm{In}_{n}^{-}$and $\mathrm{Tl}_{n}^{-}$. In contrast to $\mathrm{Al}_{n}^{-}$, where at least a part of the $\mathrm{PE}$ features (and the structures in the mass spectra) can roughly be explained by the jellium model with three valence electrons per atom, here only one electron per atom seems to contribute to the delocalized electron gas. 


\section{ACKNOWLEDGEMENTS}

We thank F. Engelke for technical support. This work has been supported by the Deutsche Forschungsgemeinschaft (DFG).

\section{REFERENCES}

I D.G. Leopold, J.H. Ho and W.C. Lineberger, J. Chem. Phys., 86 (1987) 1715.

2 O. Cheshnovsky, P.J. Brucat, S. Yang, C.L. Pettiette, M.J. Craycraft and R.E. Smalley, NATO ASI Ser B, 158 (1987) 1. C.L. Pettiette, S.H. Yang, M.J. Craycraft, J. Conceicao, R.T. Laaksonen, O. Cheshnovsky and R.E. Smalley, J. Chem. Phys., 88 (1988) 5377.

3 K. Rademann, B. Kaiser, U. Even and F. Hensel, Phys. Rev. Lett., 59 (1987) 2319.

4 M.J. Deluca, B. Niu and M.A. Johnson, J. Chem. Phys., 88 (1988) 5857.

5 G. Ganteför, M. Gausa, K.H. Mciwes-Broer and H.O. Lutz, Z. Phys. D, 9 (1988) 253.

6 G. Ganteför, M. Gausa, K.H. Meiwes-Broer and H.O. Lutz, Faraday Discuss. Chem. Soc., 86 (1988) 197.

7 K.M. McHugh, J.G. Eaton, G.H. Lee, H.W. Sarkas, L.H. Kidder, J.T. Snodgrass, M.R. Manaa and K.H. Bowen, J. Chem. Phys., 91 (1989) 3792.

8 J.L. Martins, R. Car and J. Buttet, Surf. Sci., 106 (1981) 265.

9 W. Ekardt, Phys. Rev. B, 29 (1984) 1558. W. Fkardt and Z. Penzar, Phys. Rev. B, 38 (1988) 4273. Z. Penzar and W. Ekardt, Z. Phys. D, in press.

10 W.D. Knight, K. Clemenger, W.A. de Heer, W. Saunders, M.Y. Chou and M.L. Cohen, Phys. Rev. Lett., 52 (1984) 2141.

11 T.H. Upton, Phys. Rev. Lett., 56 (1986) 2168; J. Chem. Phys., 86 (1987) 7054.

12 L.C. Balbas, A. Rubio, J.A. Alonso and G. Borstel, Chem. Phys., 120 (1988) 239.

13 V. Bonačić-Koutecký, P. Fantucci and J. Koutecký, J. Chem. Phys., 91 (1989) 3794.

14 P. Kruit and F.H. Read, J. Phys. E, 16 (1983) 313.

15 M.Y. Chou and M.L. Cohen, Phys. Lett. A, 113 (1986) 420.

16 W. Begemann, K.H. Meiwes-Broer and H.O. Lutz, Phys. Rev. Lett., 56 (1986) 2248.

17 K.J. Taylor, C.L. Pettiette, M.J. Craycraft, O. Cheshnovsky and R.E. Smalley, Chem. Phys. Lett., 152 (1988) 347.

18 R.E. Leuchtner, A.C. Harms and A.W. Castleman, J. Chem. Phys., 91 (1989) 2753.

19 K.E. Schriver, J.L. Persson, E.C. Honea and R.L. Whetten, Phys. Rev. Lett., 64 (1990) 2539.

20 D. Rayane, P. Melinon, B. Cabaud, A. Hoareau, B. Tribollet and M. Broyer, J. Chem. Phys., 90 (1989) 3295.

21 W. Begemann, S. Dreihöfer, G. Ganteför, H.R. Siekmann, K.H. Meiwes-Broer and H.O. Lutz, Elemental and Molecular Clusters, Springer Series on Materials Science, Vol. 6, Springer, Berlin, Heidelberg, 1988, p. 230.

22 D.M. Wood, Phys. Rev. Lett., 46 (1981) 749.

23 G. Ganteför, M. Gausa, K.H. Meiwes-Broer, H.O. Lutz, J. Chem. Soc. Faraday Trans., 86 (1990) 2483.

24 M. Seidel, K.H. Meiwes-Broer and M. Brack, Phys. Rev. B, in press.

25 C.E. Moore, Atomic Energy Levels, Natl. Bur. Stand. Ref. Data Ser., 35, 1971.

26 K. Clemenger, Phys. Rev. B, 32 (1985) 1359. W.A. Saunders, Thesis, Berkely, 1986. 